\title{
Metabolic reconstruction of the archaeon methanogen Methanosarcina Acetivorans
}

Vinay Satish Kumar ${ }^{1}$, James G Ferry ${ }^{2}$, Costas D Maranas ${ }^{3^{*}}$

\begin{abstract}
Background: Methanogens are ancient organisms that are key players in the carbon cycle accounting for about one billion tones of biological methane produced annually. Methanosarcina acetivorans, with a genome size of $\sim 5.7 \mathrm{mb}$, is the largest sequenced archaeon methanogen and unique amongst the methanogens in its biochemical characteristics. By following a systematic workflow we reconstruct a genome-scale metabolic model for M. acetivorans. This process relies on previously developed computational tools developed in our group to correct growth prediction inconsistencies with in vivo data sets and rectify topological inconsistencies in the model.

Results: The generated model NS941 accounts for 941 genes, 705 reactions and 708 metabolites. The model achieves $93.3 \%$ prediction agreement with in vivo growth data across different substrates and multiple gene deletions. The model also correctly recapitulates metabolic pathway usage patterns of $M$. acetivorans such as the indispensability of flux through methanogenesis for growth on acetate and methanol and the unique biochemical characteristics under growth on carbon monoxide.

Conclusions: Based on the size of the genome-scale metabolic reconstruction and extent of validated predictions this model represents the most comprehensive up-to-date effort to catalogue methanogenic metabolism. The reconstructed model is available in spreadsheet and SBML formats to enable dissemination.
\end{abstract}

\section{Background}

Genome-scale metabolic models (for recent reviews, see [1] and [2]]) are increasingly becoming available for an expanding range of organisms. There exists at least forty completed bacterial and thirteen eukaryotic metabolic reconstructions with many more under development [1]. In the past decade, several studies [3] have demonstrated a variety of uses ranging from strain optimization [4-6] pathogen drug target identification [7,8], bacterial community metabolic interactions [9] and identification of human disease biomarkers [10]. In contrast to the extensive interest devoted towards bacterial and eukaryotic metabolism reconstruction, efforts to construct archaeal metabolic models have been noticeably limited $[11,12]$. This is partly due to the current relative paucity of -omics datasets available for species from the Archaea domain. This dearth of data, however,

\footnotetext{
*Correspondence: costas@psu.edu

${ }^{3}$ Department of Chemical Engineering, The Pennsylvania State University, University Park, PA 16802, USA

Full list of author information is available at the end of the article
}

is likely to change in the near future as recent interest in methanogenic archaea has led to several sequencing efforts [13-15], as well as transcriptomic and proteomic analyses [16-20]. Furthermore, it is increasingly becoming apparent that archaeal metabolism has significant implications to the earth's climate [21] thus motivating the need to globally assess their true metabolic capabilities by reconstructing high quality metabolic models.

Methanogens (def., methane-producing) constitute the largest group of the Archaea domain of life [22]. Methanogens are terminal organisms in anaerobic microbial food chains (i.e., consortia) that break down organic matter to methane in diverse anaerobic environments in a process that helps regulate the global carbon flux [23]. The process plays a surprisingly significant role in global warming accounting for about one billion tons of the annual methane production [21,24]. Recently, Cheng and coworkers used a consortia of methanogens to convert electricity into methane thereby paving the way for converting electric current generated by renewable energy sources into renewable biofuels [25]. On the 
evolutionary front, methanogens are amongst the most ancient form of life on earth and their role as the progenitors of the first eukaryotic cell has been suggested under two separate hypotheses $[26,27]$. In addition, unique biochemical properties such as broad substrate specificity, participation of novel coenzymes in the methanogenesis pathways and the presence of unique lipids in their cell wall set these organisms apart from the bacterial and eukaryotic branches of life [28]. Therefore, the reconstruction of archaeal methanogen metabolic models could help paint a more complete picture of life's metabolic processes.

Feist and coworkers first developed a genome-scale model (named $i \mathrm{AF} 692$ ) [11] for the fresh-water methanogen, Methanosarcina barkeri using a draft version of its genome. In this paper, we reconstruct a genome-scale metabolic model for the marine methanogen, Methanosarcina acetivorans. $M$. acetivorans is an aceticlastic methanogen that was first isolated from marine microbial communities that degrade kelp into methane [29]. At over 5.7 million base pairs [15], it has the largest reported genome of all sequenced Archaea (about 20\% larger than the $M$. barkeri genome) alluding to an expanded metabolic repertoire. This repertoire includes unique methanogenic pathways, broader substrate specificity than other methanogens and a large number of duplicate genes [15,18-20,30,31]. Recent studies have shown that $M$. acetivorans uniquely exhibits both methanogenic and acetotrophic growth on carbon monoxide [31]. All these unique metabolic characteristics and planet-wide carbon balance impact $[21,24]$ provide the motivation to globally assess its metabolic capabilities.

Draft metabolic reconstructions generated using homology-based comparisons unavoidably contain some omissions and misclassifications. These errors are manifested either as unreachable metabolites or as in silico predictions that are in contrast with observed in vivo behavior [32,33]. In response to these challenges, Suthers et al., proposed a computational workflow to generate and curate the metabolic models and applied it to the metabolic reconstruction of Mycoplasma genitalium [34]. The proposed workflow makes use of two separate model correction procedures. GapFind and GapFill identify and subsequently restore connectivity to unreachable metabolites [33] and GrowMatch that reconciles in silico growth predictions with in vivo growth data [32]. In this paper, we streamline this workflow for the generation of an archaeal metabolic model and customize it to the available types of data.

We first generated a draft reconstruction of $M$. acetivorans using homology comparisons with the published model [11] of the fresh-water methanogen, M. barkeri. We then deployed a modified version of the workflow presented in Suthers et al., by combining the GapFind/
GapFill and GrowMatch steps of the procedure [34]. The completed model accounts for 941 genes, 705 reactions and 708 metabolites. The model also predicted substrate specific phenotypes of $M$. acetivorans and captured unique bioenergetics exhibited by the organism across different conditions.

\section{Results and Discussion}

The metabolic model reconstruction workflow consists of four steps. Step 1 refers to the draft model generation using bidirectional blast (BBH) and database/literature searches. Step 2 involves model modifications to ensure biomass formation for growth under all known substrates. Step 3 applies GrowMatch [32] to restore growth prediction inconsistencies and Step 4 applies GapFind and GapFill [33] to restore connectivity.

\section{Step 1: Generating Draft model}

BBH searches for each of the 692 genes included in the $i$ AF692 model were conducted against the latest genome sequence of Methanosarcina acetivorans $C 2 A$ strain [15]. At this stage of the reconstruction process, we included only open reading frames (ORFs) that have e-values (in both directions) of at most $10^{-30}$. This process yields an initial conservative model for $M$. acetivorans that has 776 genes. Based on the primary TIGR annotation of $M$. acetivorans [35] this accounts for $17.07 \%(776 / 4540)$ of all ORFs in the M. acetivorans genome. The mapping of the metabolic genotypes between these two very closely related organisms is surprisingly complex. Specifically, 369 one-to-one mappings, 1,113 one-to-many mappings and 1,050 many-tomany mappings ( $M$. barkeri to $M$. acetivorans) were generated. The large number of one-to-many and manyto-many mappings is consistent with the incidence of a high number of gene duplicates in the $M$. acetivorans genome (539 multigene families) and accounts for the additional 84 genes in $i$ VS941 over $i$ AF692 [15].

We use multiple sources to annotate the remaining 3,764 ORFs in the genome. Specifically, we preferentially assigned metabolic annotation to seven genes based on the information available at an organism-specific annotation effort for M. acetivorans [36], 51 genes based on SEED annotations [37] and 107 genes based on TIGR annotations. Interestingly out of these 165 genes as many as 68 code for isozymes. Predicted or hypothetical proteins account for the remaining 2,411 ORFs not included in the model after the annotation step. Approximately $44 \%$ of all genes in M. acetivorans (upon excluding hypotheticals and predicted proteins) were present in the draft metabolic model. The methanogenesis pathways in the $M$. acetivorans model were modified to account for known differences documented in the literature. 
Specifically, $M$. acetivorans and $M$. barkeri use different electron transport chains to generate ATP when they grow on acetate. The electron transport chain in $M$. barkeri consists of a pair of hydrogenases, Ech and Vho that couple hydrogen production/oxidation to proton translocation outside the membrane [30]. In $M$. acetivorans, $\mathrm{ECH}$ and $\mathrm{VHO}$ are absent and instead it is hypothesized that an electron transfer complex Rnf (abbreviation in $i$ VS941: RNF) oxidizes reduced ferredoxin to generate a sodium ion gradient which is then exchanged for a proton gradient by the multiple resistance/pH regulation $\mathrm{Na}+/ \mathrm{H}+$ antiporter (abbreviation in iVS941: MRP) [30]. M. acetivorans grows on carbon monoxide as a substrate in the absence of hydrogen using both the electron transport chain (the methanogenic (methane forming) pathway) and substrate level phosphorylation (acetogenic (acetate forming) pathway). Alternatively, it has been proposed that $M$. barkeri grows on $\mathrm{CO}$ only in the presence of hydrogen and oxidizes $\mathrm{CO}$ to $\mathrm{CO}_{2}$ and uses the resulting energy to produce hydrogen that is then reoxidized using the hydrogenases (discussed above) to produce electrons needed to reduce $\mathrm{CO}$ to methane [30]. On C1 compounds such as methanol and methylamines, both organisms have a methylotrophic pathway that disproportionates the carbon to form carbon dioxide and methane [38]. Interestingly, one mole of substrate is oxidized to generate reducing equivalents required to produce three moles of methane.

In contrast with other archaeal models [11,12], we delineated methyltransferase specificity $[39,40]$ for different substrates of $M$. acetivorans . We also generated the Gene-Protein-Reaction mappings for the $M$. acetivorans model using as a starting point the $i$ AF692 model based on the AUTOGRAPH method developed by Notebaard and coworkers [41]. All exchange reactions and nongene associated intracellular reactions available in the $i$ AF692 model were also appended to the model, as we did not find any evidence to the contrary [see Methods]. Upon conclusion of Step 1, a draft model with 941 genes, 705 reactions and 708 metabolites was generated.

\section{Step 2: Model correction to enable biomass formation}

We determine the metabolic capabilities of the assembled draft model to grow on known methanogenic substrates by first specifying the biomass equation and then specifying the composition of the minimal medium. The first requirement is addressed by assuming that the set of components that compose the biomass equation in $M$. acetivorans is identical to the one used in the $i$ AF692 model. The stoichiometric coefficients of the nucleotide components (datp, dgtp, dctp, dttp, ctp and gtp) were modified to reflect the difference in the $\mathrm{G} / \mathrm{C}$ contents of the two organisms (see Additional File 1).
The utilization of the same biomass component set is supported by experimental data on the minimal medium (Ferry et al., unpublished data). The minimal growth medium contained six additional vitamins and trace elements (pyridoxine-HCL, sodium molybdate, thioctic acid, nitrilo tri acetic acid and boric acid) over the one used in $i$ AF692 [11]. We chose to exclude them from our model as no metabolic role for them was identified based on literature searches or gleaned by the model.

Equipped with the biomass composition and the minimal medium, we tested the capability of the draft model to enable growth on the following known methanogenic substrates: carbon monoxide, acetate, methanol and monomethylamine, dimethylamine and trimethylamine [29]. The draft model did not exhibit growth on any of these substrates motivating the use of GapFind [33] to identify the biomass precursor metabolites that could not be produced using these substrates in a minimal medium. GapFind revealed that the same precursor metabolite Adenosylcobalamin-HBI could not be produced for all substrate choices in the draft model. We used GapFill [33] to restore flow through this metabolite. This was achieved under all aforementioned substrate conditions by adding an exporter for the cofactor, tetrahydrosarcinapterin. While the export of the cofactor could be an in silico response to an imbalance of cofactors and there is no evidence in the literature for the presence of a tetrahydrosarcinapterin exporter, it is possible that an enzyme outside the cell wall may utilize the cofactor as a substrate.

\section{Step 3: Evaluating and improving model performance using GrowMatch}

After ensuring in silico growth on a defined medium across different substrates, we further examined the model by testing for growth prediction agreement with experimental data across different genetic/environmental perturbations. Using literature searches, we assembled a dataset consisting of in vivo growth data for 60 different conditions (See Table 1). As shown in Table 1, growth data was assembled for eighteen genetic perturbations for growth on methanol, thirteen on acetate as carbon substrates, nine on carbon monoxide as carbon and energy source, and 20 on methylamines as carbon substrates. Not surprisingly, most of these gene deletions are in methanogenesis pathways (Table 1) indicative of the significant attention this pathway has received before.

In line with previous approaches [42] the growth cutoff for classifying a mutant as a "Growth" or a "NoGrowth" mutant was chosen to be $1 / 3^{\text {rd }}$ of average growth across the dataset. Using this cutoff and the terminology introduced in the GrowMatch procedure [32] we arrive at 43 GG (mutant exhibits in silico and in vivo 
Table 1 In vivo gene deletion data used evaluate and improve iVS941's predictive capabilities (Citations are indicated in square brackets)

\begin{tabular}{|c|c|c|c|c|c|c|}
\hline \multirow[b]{2}{*}{ Gene deletions } & \multicolumn{6}{|c|}{ Substrate } \\
\hline & acetate & carbon monoxide & methanol & monomethylamine & dimethylamine & trimethylamine \\
\hline ackR & NGNG & GNG[51] & - & - & - & - \\
\hline ATP synthase & NGNG[52] & - & GNG[52] & - & - & - \\
\hline DMTsD & GG[53] & GG[53] & GG[53] & - & - & GG[53] \\
\hline$m t s D+m t s F$ & GG[53] & GG[53] & GG[53] & - & - & GG[53] \\
\hline$m t s D+m t s H$ & GG[53] & GG[53] & GG[53] & - & - & GG[53] \\
\hline $\mathrm{mtsF}$ & GG[53] & GG[53] & GG[53] & - & - & GG[53] \\
\hline $\mathrm{mtsH}$ & GG[53] & GG[53] & GG[53] & - & - & GG[53] \\
\hline$m t s F+m t s H$ & GG[53] & GG[53] & GG[53] & - & - & GG[53] \\
\hline lysK & - & - & GG[54] & - & GG[54] & GG[54] \\
\hline lysS & - & - & GG[54] & GG[54] & GG[54] & GG[53] \\
\hline MCR & NGNG[43] & GNG[43] & NGNG[43] & GNG[43] & GNG[43] & GNG[43] \\
\hline mtaA1 & - & - & NGNG[39] & & - & - \\
\hline mtaA1 + MT1 & GNG[39] & - & - & - & - & - \\
\hline mtaA2 & - & - & GG[39] & - & - & - \\
\hline mtaCB1 & - & - & GG[55] & - & - & - \\
\hline$m t a C B 1+m t a C B 2$ & - & - & GG[55] & - & - & - \\
\hline$m t a C B 1+m t a C B 2+m t a C B 3$ & - & - & GNG[55] & - & - & - \\
\hline $\mathrm{mtaCB} 2$ & - & - & GG[55] & - & - & - \\
\hline mtaCB3 & - & - & GG[55] & - & - & - \\
\hline mtbA & - & - & - & NGNG[39] & NGNG[39] & - \\
\hline$m t b A$ & - & - & - & - & - & GG[54] \\
\hline ppylT & GG[56] & & GG[56] & GNG[56] & GNG[56] & GNG[56] \\
\hline ptaR & NGNG & GNG[51] & - & - & - & - \\
\hline Rnf complex & GNG[57] & - & - & - & - & - \\
\hline
\end{tabular}

"Growth") thirteen GNG (mutant exhibits in silico "Growth" and in vivo "No-Growth") and eight NGNG (mutant exhibits in silico and in vivo "No-Growth") cases. Notably, the incidence of only GNG model/ experimental discrepancies indicates that the $\mathrm{draft}$ model tends to over-predict the metabolic capabilities of the organism when in error. A closer examination reveals that in 31 out of 43 GG cases the deleted genes correspond to isozymes while six correspond to deletions of methyltransferases. In all these cases both the model and the experiment agree that the deleted genes are non-essential. Of the nine GNG cases that could be resolved, eight were resolved by conditionally suppressing one additional reaction and one was resolved by carrying out a single global suppression (global suppressions do not affect any consistent GG cases when carried out globally whereas conditional suppressions affect atleast one GG case)(see Additional File 1).

Figure 1 highlights two examples of GrowMatch's resolution of GNG mutants in M. acetivorans. As shown in Figure $1(\mathrm{~A})$, the genes encoding for Methyl-coenzyme $M$ reductase (MCR) (the reaction that forms methane) under growth on Carbon Monoxide are non-essential in silico and essential in vivo [43]. GrowMatch suggests suppressing either the reaction catalyzed by acetate kinase (ACKr) or phosphotransacetylase (PTAr) to restore consistency to this mutant. These hypotheses are consistent with the bioenergetics when $M$. acetivorans grows on $\mathrm{CO}$ as the sole energy source [31]. Both the acetogenic and methanogenic branches of the methanogenesis pathway are energy yielding. Flux in the methanogenic branch results in a proton and sodium ion gradient which is then used to synthesize ATP catalyzed by the proton translocating ATP synthase. Alternatively, flux through the acetogenic branch results in ATP synthesis using substrate level phosphorylation when acetyl phosphate is converted to acetate by acetate kinase. When Methyl-coenzyme $M$ reductase is deleted there is no mechanism to recycle HS-CoM for another round of methylation and the Mtr-catalyzed methyl transfer reaction (methyl-THSPT:CoM-SH methyltransferase) coupled to generation of the sodium gradient is also deactivated thereby diverting methyl tetrahydrosarcinapterin $\left(\mathrm{CH}_{3}-\right.$ THSPT) towards synthesis of acetate and ATP. Therefore suppressing ACKr (or equivalently PTAr) in a mutant lacking Methyl coenzyme reductase (and consequently, the methane forming branched pathway) ensures that both energy yielding pathways are deactivated thereby halting growth. 

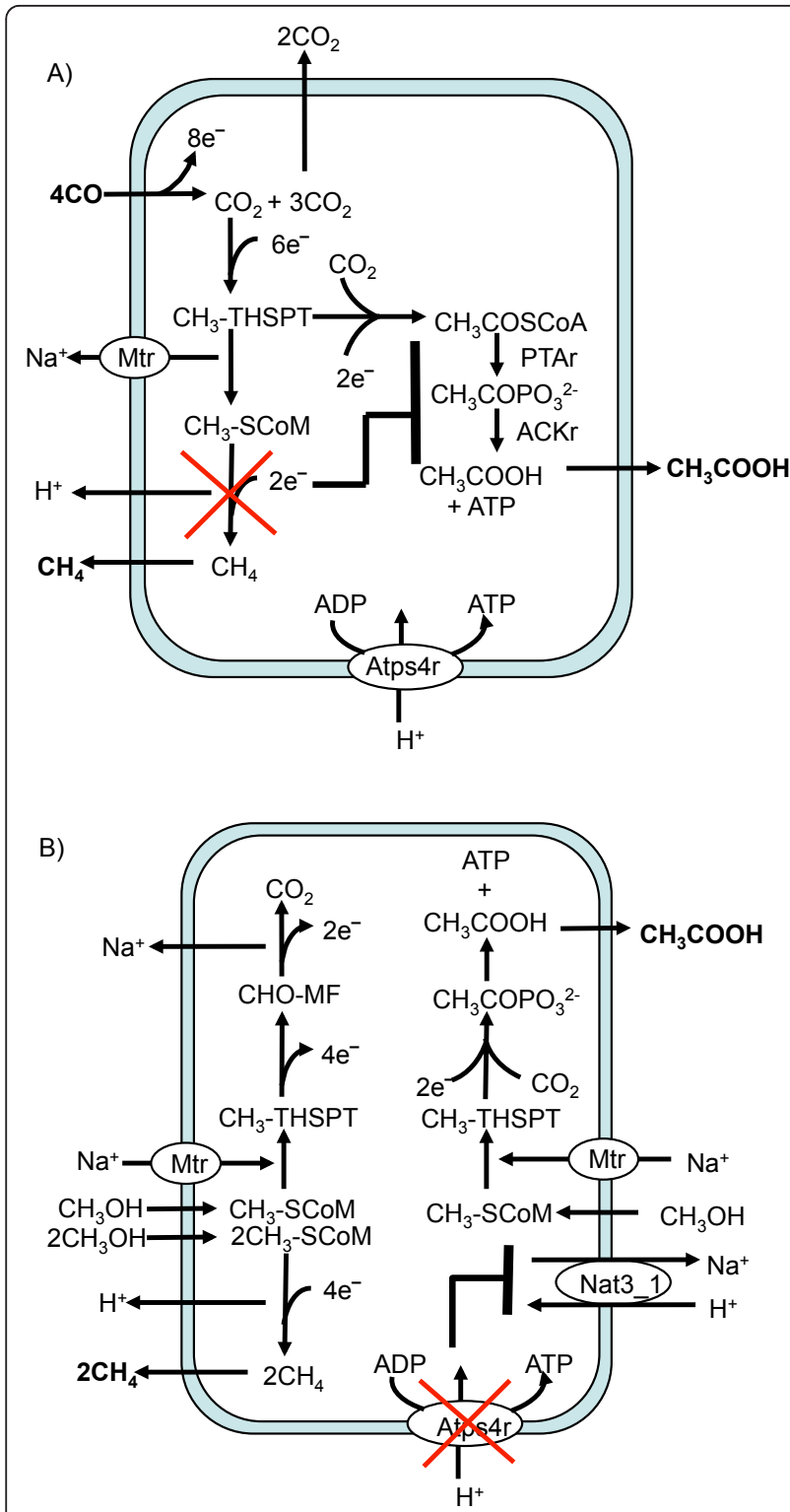

Figure 1 Resolving GNG mutants using GrowMatch. A) GrowMatch resolution of the GNG mutant characterized by deleting Methyl Coenzyme Reductase with carbon monoxide as the carbon source. B) GrowMatch's resolution of the GNG mutant characterized by deleting ATP Synthase with methanol as the carbon source.

In the second case (Figure 1(B)), deleting ATP synthase results in a GNG mutant when the organism grows on methanol as the sole carbon and energy source [19]. This deletion negates proton-coupled generation of energy via methanogenesis leaving substrate level generation of energy via acetogenesis. GrowMatch suggests restoring consistency to this mutant by suppressing the sodium proton antiporter (abbreviation in iVS941: Nat3_1). Suppressing this reaction in this mutant metabolic network deactivates flux in the sodium-dependent reaction methyl-
THSPT:coenzyme M methyltransferase (abbreviation in $i$ VS941: MTSPCMMT) which results in no flux in the acetogenesis pathway (Figure 1B)).

\section{Step 4: Network connectivity analysis and restoration}

After evaluating and improving the model using in vivo gene deletion data, we used the automated procedures GapFind and GapFill [33] to identify and rectify any remaining network connectivity inconsistencies. Using GapFind, we identify 92 metabolites (i.e., $13.1 \%$ of all metabolites in model) that cannot be produced for any choice of carbon substrate. Not surprisingly, none of the 95 no production metabolites were present in the methanogenesis pathway alluding to the completeness of its reconstruction. Interestingly, of the 161 metabolites present in the $M$. acetivorans model but absent in $i$ AF692, 101 can be produced whereas 60 have blocked production routes. Notably, GapFind revealed that 35 out of these 95 metabolites could also not be produced in the $i$ AF692 model of $M$. barkeri. Flow restoration to all 95 metabolites was attempted using GapFill by adding reactions from KEGG [44]. In this step, we restored consistency to only two of the 92 no production metabolites. Flow through these metabolites was restored by treating two existing reactions (cob(I)alamin-HBI adenosyltransferase and hydroxyethylthiazole kinase) as reversible.

\section{Model characteristics for iNS941}

Table 2 contrasts the model statistics for the $i$ VS 941 model against previously constructed archaeal models. Most reaction entries in $i$ VS941 model are associated with very stringent e-values implying a high confidence for their inclusion. Furthermore, the inclusion of seven regulatory constraints that allow for substrate specific activation of methyltransferases and the addition of reactions using multiple pieces of evidence are unique features of this model. Finally, in contrast to the remaining models, the iVS941 model documents global and conditional suppressions based on systematic evaluation of model predictions with in vivo growth data and network gap correction.

We compared flux values through the methane forming reaction catalyzed by Methyl Coenzyme Reductase and the biomass equation to ascertain the extent of coupling between flux in the methanogenesis pathway and in silico growth rates. We identified the range of methane production flux by maximizing and minimizing flux through the MCR reaction for different values of biomass formation. Conversely, we identified the range of biomass production for different values of required methane production. Figure 2 shows these plots for growth on methanol, acetate and carbon monoxide.

As shown in Figure 2(A) and 2(B), a positive biomass flux implies a non-zero MCR flux for growth in methanol 
Table 2 Comparison between iVS941 and other available Archaeal models

\begin{tabular}{ccccc}
\hline & Methanosarcina acetivorans & Methanosarcina barkeri & Halobacterium salinarum & Methanococcus jannaschii \\
\hline Genome size & $5.7 \mathrm{Mb}$ & $4.8 \mathrm{Mb}$ & $2.7 \mathrm{Mb}$ & $1.7 \mathrm{Mb}$ \\
ORF's & 4540 & 3680 & 2867 & 1792 \\
Metabolic genes & 941 & 692 & 490 & 436 \\
Unique proteins & 941 & 542 & 490 & 266 \\
Reactions & 705 & 619 & 708 & 609 \\
gene-associated & 590 & 509 & 568 & 297 \\
non gene-associated & 115 & 110 & 133 & 312 \\
transport reactions & & 88 & 111 & 1 \\
Metabolites & 708 & 558 & 557 & 510 \\
Gaps & 93 & 35 & & -
\end{tabular}

and acetate but not the reverse. Using the terminology introduced in [45], this implies that the flux in biomass reaction is directionally coupled to the flux in MCR. This is consistent with the indispensability of the methanogenic branch when $M$. acetivorans grows on acetate and methanol $[30,43]$. Moreover, the maximum biomass formation is reached at when the flux through MCR is fixed at $74 \%$ of its maximum value for growth on methanol and $86 \%$ for growth on methanol. At maximum biomass production, the ratio of biomass to methane production is 0.016 GDW/mmol and $0.005 \mathrm{GDW} / \mathrm{mmol}$ for growth on methanol and acetate, respectively. This higher biomass yield is qualitatively consistent with the higher energetic yield per mole of methanol observed for M. acetivorans [46].

Figure 2(C) illustrates the predictions of the $i$ VS941 model for growth on carbon monoxide as the sole carbon and energy substrate. The model prediction that the methanogenic branch is dispensable when $M$. acetivorans grows on carbon monoxide is consistent with the mechanism proposed in [26,31]. The proposed mechanism hypothesizes alternate means of ATP generation (electron transport chain: methanogenic branch or substrate level phosphorylation: acetogenic branch) when $M$. acetivorans grows on carbon monoxide. Notably, the maximum biomass production is achieved at $58 \%$ of the maximum flux in the MCR reaction and the ratio of the two fluxes is $0.033 \mathrm{GDW} / \mathrm{mmol}$. It has been previously established that the acetogenic and methanogenic branches of the pathway are energy yielding when M. acetivorans grows on carbon monoxide [31]. Using the coupling analysis described above, we find that the acetogenic and methanogenic branches are not coupled This supports the independence of the energy yielding branches for growth on carbon monoxide.

\section{Conclusions}

Metabolic reconstruction technology has been used extensively to document the metabolic repertoire of organisms in the Bacteria and the Eukarya domains [47]. Here, we take advantage of the increased availability of experimental datasets and genomic information for archaeal organisms to build the metabolic model, called $i \mathrm{VS} 941$, of the archaeon with the largest known genome, Methanosarcina acetivorans. The $i$ VS941 model is constructed using a systematic procedure that enables sequential evaluation and improvement of model capabilities. The model consists of 705 reactions, 708 metabolites and 941 genes; the latter accounting for about $44 \%$ of all ORFs in $M$. acetivorans with putative annotations [35]. The completed model has metabolites (87\%) that can be produced and it has a high agreement of $93.3 \%$ against published in vivo growth data across environmental and genetic perturbations (thirty data points) with specificity of $81 \%$ (i.e., percent of correctly identified essential genes) and selectivity of $89.7 \%$ (i.e., percent of correctly identified non-essential genes). Additionally, the model recapitulates substrate-specific energetic characteristics such as ATP synthase indispensability for growth on acetate/methanol and its dispensability for growth on carbon monoxide.

The number of reactions included in the draft model under Step 1 is quite sensitive to the adopted BLAST cutoff. The number of gene entries increases to 1,090 when the cutoff is relaxed to $10^{-20}$ from the 776 entries for the adopted cutoff of $10^{-30}$. This more stringent cutoff was chosen to ensure that the draft model did not contain any falsely added functionalities. We have found that it is much easier to find and add missing functionalities than correctly identifying and removing erroneous ones. Interestingly, all but one reaction in the methanogenesis pathway known to occur in $M$. acetivorans were included in the draft model using the most stringent cutoff. Reaction ECH Hydrogenase which is known to occur in $M$. barkeri but not in $M$. acetivorans was excluded from the draft model. 


\section{A) Growth on methanol}
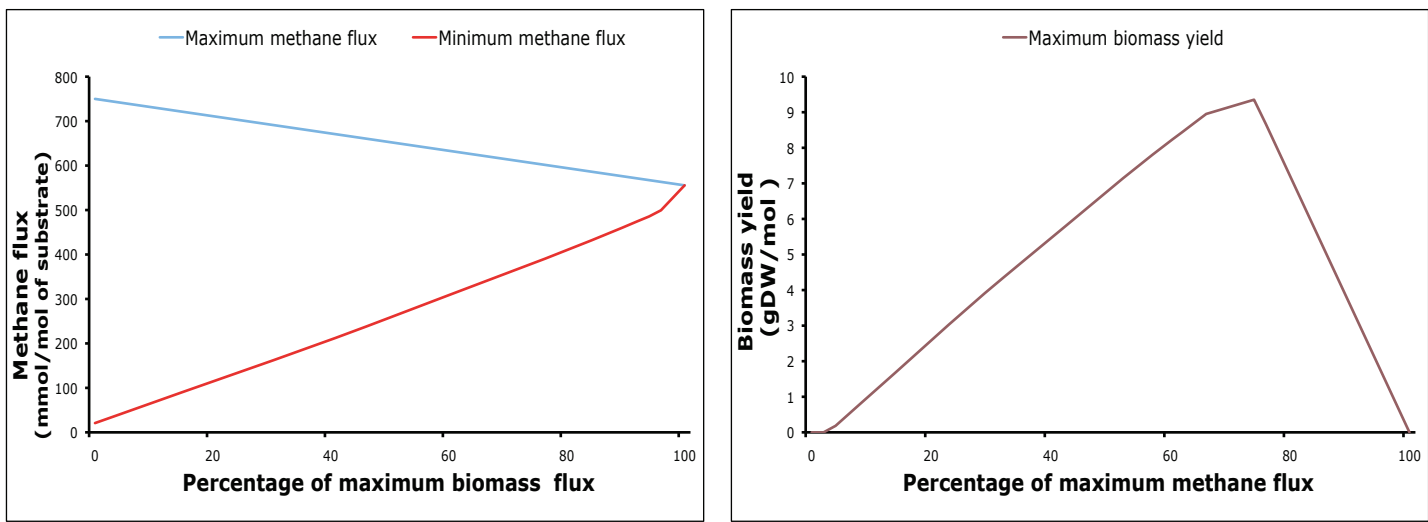

\section{B) Growth on acetate}
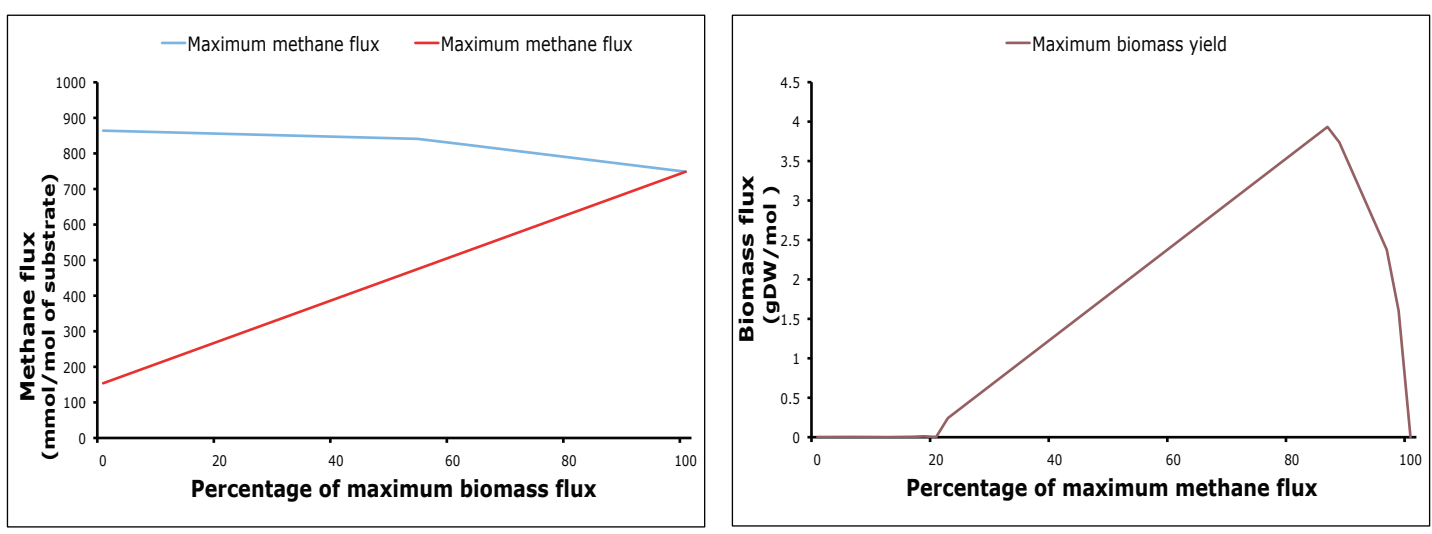

\section{C) Growth on carbon monoxide}
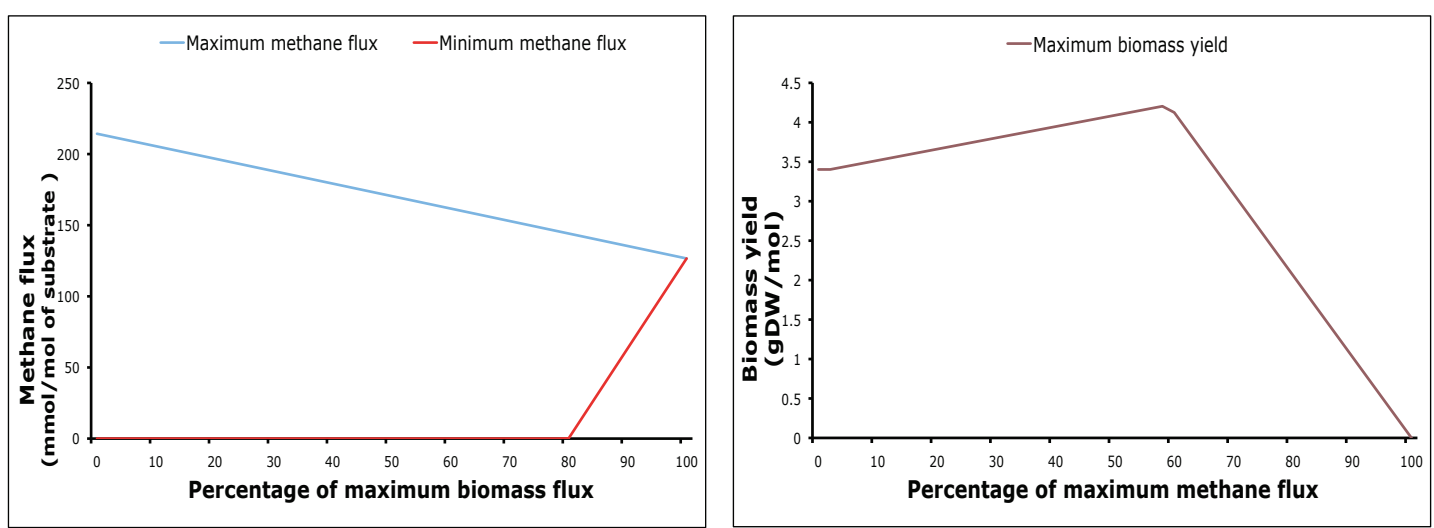

Figure 2 Flux coupling analysis between yield in Methyl coenzyme reductase and biomas yield on A) methanol, B) acetate, C) carbon monoxide. All values of yields in $\mathrm{mmol} / \mathrm{gDW} \mathrm{hr}{ }^{-1}$ and are normalized to the respective substrate uptake rates fixed at $1000 \mathrm{mmol} / \mathrm{gDW} \mathrm{hr}$.

This constructed $i$ VS941 model represents the most comprehensive up-to-date effort to catalogue methanogenic metabolism. Given the attention methanogenic consortia have received and the growing amount of metagenomic data [48], this model can be used to assess the biological impact on carbon balance of methanogenic communities. This organism-specific compilation of the metabolic repertoire of $M$. acetivorans can serve as the framework for fusing additional experimental data on methanogens as they become 
available. The model is available in SBML format to enable dissemination (Additional File 2).

\section{Methods}

\section{Generation of initial model}

We generate the initial model for $M$. acetivorans by taking advantage of an existing genome-scale metabolic model for the marine methanogen M. barkeri (iAF692). The iAF692 model is based on a draft version the $M$. barkeri fusaro genome [11]. We first mapped the genes from iAF692 onto the current genome-sequence of $M$. barkeri to restore consistency with the most upto-date genomic information. For every gene in the $i$ AF692 model, we retrieved the corresponding protein sequence (personal communication with Adam Feist of UCSD) and conducted bidirectional BLAST (BBH) (BLASTp [49]) searches against the current genome sequence of $M$. barkeri.

The draft reconstruction for $M$. acetivorans is generated by conducting bidirectional BLAST (BLASTp) searches for each one of the 692 genes in $i$ AF692 against its genome and including only those genes/protein/reaction associations with an e-value of better than $10^{-30}$. We used multiple sources to annotate the remaining genes in $M$. acetivorans in the following order. The primary resource was an ongoing effort at the University of Maryland (carried out in the Sowers $\mathrm{Lab}$ at the Center for Marine Biotechnology). Whenever such information was lacking we alternatively relied on first the SEED database [37] and finally the TIGR [35] annotations.

Upon obtaining annotations for the remaining genes, we pinpointed metabolic genes by searching each annotation against the KEGG ligand [44] database and retrieving corresponding reactions. KEGG reactions are not necessarily charge/mass balanced. We manually checked the reactions we added and found that reactions involving tRNA molecules were not mass balanced. For annotations with no synonyms in the KEGG ligand database, we use their Enzyme Commission Number (if available) to search the Swiss-Prot database [50] and retrieve the metabolic reaction(s) (if at all) they are associated with. Finally, we also included reactions that are known to be present in $M$. acetivorans but absent in $M$. barkeri (e.g., reactions for CO metabolism. We use the AUTOGRAPH procedure developed by Notebaard et al., to generate the gene-protein-reaction (GPR) associations [41]. This procedure uses bidirectional BLAST hits (BBH) to generate GPR's for new metabolic reconstructions ( $M$. acetivorans in our case) using the GPR's of related metabolic models ( $M$. barkeri). We also added non-gene associated reactions and exchange reactions in $i$ AF692 to the model unless we found evidence to contrary.
Model fidelity improvement using available data sources

Upon the generation of the draft model the next step involves the systematic elimination of network gaps using GapFind/GapFill [33] and growth prediction inconsistencies using GrowMatch [32]. These procedures are deployed in a synergistic manner to provide mutually corroborating evidence for model correction.

\section{Step 1}

We generate the draft model as discussed above.

\section{Step 2}

We test the ability of the model to grow on known substrates. If it doesnt, we use modified versions of GapFind and GapFIll respectively to identify biomass precursors that cannot be produced and ensure their production. We allow for addition of functionalities at this step only if the BLAST e-value is lower than $10^{-2}$. Upon completion of this step all biomass components are available in $i$ VS941.

\section{Step 3}

We compare in silico biomass production in $i$ VS941 against available in vivo growth data across different environmental/genetic perturbations. Mutants are classified as Grow/Grow (GG), No-Grow/Grow (NGG), Grow/ No-Grow (GNG) and No-Grow/No-Grow (NGNG) following the definitions proposed in [32]. GNG mutants are resolved by identifying global/conditional suppressions in the $i$ VS941 network using GrowMatch. Upon completion of this step, all in silico/in vivo growth inconsistencies that could be corrected by either removing or adding reactions available in databases resolved.

\section{Step 4}

We next identified metabolites that cannot be produced or consumed using GapFind. Using GapFill, we restore connectivity to them by appending only reactions that have $\mathrm{BBH}$ e-values of less than $10^{-10}$.

In addition, we mined for all published articles having the word "Acetivorans" anywhere in their content in the Web of Science and PubMed databases and download these articles using EndNote ${ }^{\mathrm{Web}}$. We used the mdfind command on a MacBook ${ }^{\mathrm{TM}}$, search for articles that have loci-names of $M$. acetivorans genes included in the $i$ VS941 Model. This enables a relatively quick search for literature evidence supporting (or not) annotations in the $i$ VS941 Model. We update the model to resolve any incorrect annotations identified in this step and consolidate information from articles not included in the above search domain but have information regarding methanogenesis [39].

\section{Additional material}

Additional file 1: The iVS941 model in spreadsheet format.

Additional file 2: The iVS941 model in SBML format. 


\section{Acknowledgements}

The authors would like to thank Dr. Anthony Burgard and Dr Patrick Suthers for valuable discussions. The authors gratefully acknowledge funding from the DOE grant (DE-FG03 01ER25499).

\section{Author details}

'Joint BioEnergy Institute, 5885 Hollis street, Emeryville, California 94608, USA. ${ }^{2}$ Department of Biochemistry and Molecular Biology, The Pennsylvania State University, University Park, PA 16802, USA. ${ }^{3}$ Department of Chemical Engineering, The Pennsylvania State University, University Park, PA 16802, USA.

\section{Authors' contributions}

CDM conceived the study. VSK built the model and performed the computational analyses. VSK, JGF and CDM drafted the manuscript. All authors read and approved the final version of the manuscript.

Received: 1 March 2010 Accepted: 15 February 2011

Published: 15 February 2011

\section{References}

1. Feist $A M$, et al: Reconstruction of biochemical networks in microorganisms. Nat Rev Microbiol 2009, 7(2):129-43.

2. Park JM, Kim TY, Lee SY: Constraints-based genome-scale metabolic simulation for systems metabolic engineering. Biotechnol Adv 2009, 27(6):979-88.

3. Feist AM, Palsson BO: The growing scope of applications of genome-scale metabolic reconstructions using Escherichia coli. Nat Biotechnol 2008, 26(6):659-67.

4. Burgard AP, Pharkya p, Maranas CD: Optknock: a bilevel programming framework for identifying gene knockout strategies for microbial strain optimization. Biotechnol Bioeng 2003, 84(6):647-57.

5. Pharkya P, Burgard AP, Maranas CD: Exploring the overproduction of amino acids using the bilevel optimization framework OptKnock. Biotechnol Bioeng 2003, 84(7):887-99.

6. Hatzimanikatis $V$, et al: Application of mathematical tools for metabolic design of microbial ethanol production. Biotechnol Bioeng 1998, 58(23):154-61.

7. Lee DS, et al: Comparative genome-scale metabolic reconstruction and flux balance analysis of multiple Staphylococcus aureus genomes identify novel antimicrobial drug targets. J Bacteriol 2009, 191(12):4015-24.

8. Pinney JW, et al: Metabolic reconstruction and analysis for parasite genomes. Trends Parasitol 2007, 23(11):548-54.

9. Stolyar $\mathrm{S}$, et al: Metabolic modeling of a mutualistic microbial community. Mol Syst Biol 2007, 3:92.

10. Shlomi T, Cabili MN, Ruppin E: Predicting metabolic biomarkers of human inborn errors of metabolism. Mol Syst Biol 2009, 5:263.

11. Feist $\mathrm{AM}$, et al: Modeling methanogenesis with a genome-scale metabolic reconstruction of Methanosarcina barkeri. Mol Syst Biol 2006, 2:2006-0004

12. Gonzalez $\mathrm{O}$, et al: Reconstruction, modeling \& analysis of Halobacterium salinarum R-1 metabolism. Mol Biosyst 2008, 4(2):148-59.

13. Maeder $\mathrm{DL}$, et al: The Methanosarcina barkeri genome: comparative analysis with Methanosarcina acetivorans and Methanosarcina mazei reveals extensive rearrangement within methanosarcinal genomes. $J$ Bacteriol 2006, 188(22):7922-31.

14. Deppenmeier $\mathrm{U}$, et al: The genome of Methanosarcina mazei: evidence for lateral gene transfer between bacteria and archaea. J Mol Microbiol Biotechnol 2002, 4(4):453-61,

15. Galagan JE: The Genome of M. acetivorans Reveals Extensive Metabolic and Physiological Diversity. Genome Research 2002, 12(4):532-542.

16. Rother M, Oelgeschläger E, Metcalf WW: Genetic and proteomic analyses of $\mathrm{CO}$ utilization by Methanosarcina acetivorans. Archives of Microbiology 2007.

17. SabrinaTachdjian K, Conners S: Functional Genomics of Stress Response in Extremophilic Archaea. Archaea: New Models for Prokaryotic Biology 2008.

18. Li L, et al: Quantitative Proteomic and Microarray Analysis of the Archaeon MethanosarcinaacetivoransGrown with Acetate versus Methanol. Journal of Proteome Research 2007, 6(2):759-771.
19. Li Q, et al: Proteome of Methanosarcinaacetivorans Part II: Comparison of Protein Levels in Acetate- ... Journal of Proteome Research 2005.

20. Li Q, et al: Proteome of Methanosarcinaacetivorans Part I: An Expanded View of the Biology of the Cell. Journal of Proteome Research 2005.

21. Schlesinger WH: Biogeochemistry: an analysis of global change. San Diego, Calif:. Academic Press; 2 1997, xiii, 588 p., [2] p. of plates.

22. Ferry JG: Methanogenesis: ecology, physiology, biochemistry \& genetics. Chapman \& Hall microbiology series New York: Chapman \& Hall; 1993, x, 536 p.

23. Mclnerney MJ, Sieber JR, Gunsalus RP: Syntrophy in anaerobic global carbon cycles. Curr Opin Biotechnol 2009, 20(6):623-32.

24. Bloom AA, et al: Large-scale controls of methanogenesis inferred from methane and gravity spaceborne data. Science 327(5963):322-5.

25. Cheng $S$, et al: Direct biological conversion of electrical current into methane by electromethanogenesis. Environ Sci Technol 2009, 43(10):3953-8.

26. Ferry J, House C: The stepwise evolution of early life driven by energy conservation. Molecular biology and evolution 2006, 23(6):1286-1292.

27. Battistuzzi FU, Feijao A, Hedges SB: A genomic timescale of prokaryote evolution: insights into the origin of methanogenesis, phototrophy, and the colonization of land. BMC Evol Biol 2004, 4:44.

28. Ferry JGKK, ed: Methanogenesis. In Archaea: Molecular Cell Biology. Volume 7. Edited by: Cabicchioli R. ASM Press; 2007.

29. Sowers K, Baron S, Ferry J: Methanosarcina acetivorans sp. nov., an acetotrophic methane-producing bacterium .... Applied and Environmental Microbiology 1984

30. Ferry JG, Lessner DJ: Methanogenesis in Marine Sediments. Annals of the New York Academy of Sciences 2007, 1125(1):147-157.

31. Lessner $\mathrm{D}$, et al: ... of $\mathrm{CO} 2$ to methane in CO-grown Methanosarcina acetivorans revealed by proteomics. Proceedings of the National Academy of Sciences 2006.

32. Kumar VS, Maranas CD: GrowMatch: an automated method for reconciling in silico/in vivo growth predictions. PLoS Comput Biol 2009, 5(3):e1000308.

33. Satish Kumar V, Dasika MS, Maranas CD: Optimization based automated curation of metabolic reconstructions. BMC bioinformatics 2007, 8:212.

34. Suthers PF, et al: A genome-scale metabolic reconstruction of Mycoplasma genitalium, iPS189. PLoS Comput Biol 2009, 5(2):e1000285.

35. Comprehensive Microbial Resource. [http://cmr.jcvi.org/tigr-scripts/CMR/ CmrHomePage.cgi].

36. Sowers K: [http://carb.umbi.umd.edu/g2f/].

37. Overbeek $R$, et al: The subsystems approach to genome annotation and its use in the project to annotate 1000 genomes. Nucleic Acids Res 2005 33(17):5691-702.

38. Ferry JG: Enzymology of one-carbon metabolism in methanogenic pathways. FEMS Microbiol Rev 1999, 23(1):13-38.

39. Bose A, Pritchett MA, Metcalf WW: Genetic Analysis of the Methanol- and Methylamine-Specific Methyltransferase 2 Genes of Methanosarcina acetivorans C2A. Journal of Bacteriology 2008, 190(11):4017-4026.

40. Ding $Y$, et al: ... system that are differentially expressed in methanol-and acetate-grown Methanosarcina ... FEMS Microbiology Letters 2002.

41. Notebaart RA, et al: Accelerating the reconstruction of genome-scale metabolic networks. BMC bioinformatics 2006, 7:296.

42. Joyce $A R$, et al: Experimental and computational assessment of conditionally essential genes in Escherichia coli. J Bacteriol 2006, 188(23):8259-71

43. Rother $M$, et al: ... methyl-coenzyme $M$ reductase is essential in Methanosarcina acetivorans C2A and allows ... Journal of Bacteriology 2005.

44. Kanehisa $\mathrm{M}$, et al: KEGG for linking genomes to life and the environment. Nucleic Acids Res 2008, , 36 Database: D480-4.

45. Burgard AP, et al: Flux coupling analysis of genome-scale metabolic network reconstructions. Genome Res 2004, 14(2):301-12.

46. Thauer R, et al: Methanogenic archaea: ecologically relevant differences in energy conservation. Nature Reviews Microbiology 2008.

47. Thiele I, Palsson BO: A protocol for generating a high-quality genomescale metabolic reconstruction. Nat Protoc 5(1):93-121.

48. Pernthaler A, et al: Diverse syntrophic partnerships from deep-sea methane vents revealed by direct cell capture and metagenomics. Proc Natl Acad Sci USA 2008, 105(19):7052-7. 
49. Altschul SF, et al: Gapped BLAST and PSI-BLAST: a new generation of protein database search programs. Nucleic Acids Res 1997 25(17):3389-402.

50. Bairoch $A$, et al: The Universal Protein Resource (UniProt). Nucleic Acids Res 2005, 33 Database: D154-9.

51. Rother M, Metcalf W: Anaerobic growth of Methanosarcina acetivorans C2A on carbon monoxide: An unusual way of life for a methanogenic archaeon. Proceedings of the National Academy of Sciences 2004, 101(48):16929-16934

52. Li L, et al: ... and Microarray Analysis of the Archaeon Methanosarcina Acetivorans Grown with Acetate ... Journal of Proteome Research 2007.

53. Oelgeschlager $E$, Rother $M$ : In vivo role of three fused corrinoid/methyl transfer proteins in Methanosarcina acetivorans. Molecular Microbiology 2009.

54. Mahapatra A, et al: Class I and class II lysyl-tRNA synthetase mutants and the genetic encoding of pyrrolysine in Methanosarcina spp. Molecular Microbiology 2007, 64(5):1306-1318.

55. Pritchett MA, Metcalf WW: Genetic, physiological and biochemical characterization of multiple methanol methyltransferase isozymes in Methanosarcina acetivorans C2A. Molecular Microbiology 2005, 56(5):1183-1194

56. Mahapatra A, et al: Characterization of a Methanosarcina acetivorans mutant unable to translate UAG as pyrrolysine. Molecular Microbiology 2006, 59(1):56-66.

57. Ferry JG, Lessner DJ: Methanogenesis in marine sediments. Ann N Y Acad Sci 2008, 1125:147-57.

doi:10.1186/1752-0509-5-28

Cite this article as: Satish Kumar et al:: Metabolic reconstruction of the archaeon methanogen Methanosarcina Acetivorans. BMC Systems Biology 2011 5:28.

\section{Submit your next manuscript to BioMed Central and take full advantage of:}

- Convenient online submission

- Thorough peer review

- No space constraints or color figure charges

- Immediate publication on acceptance

- Inclusion in PubMed, CAS, Scopus and Google Scholar

- Research which is freely available for redistribution

Submit your manuscript at www.biomedcentral.com/submit 